\title{
Risk-benefit assessment of ivabradine in the treatment of chronic heart failure
}

This article was published in the following Dove Press journal:

Drug, Healthcare and Patient Safety

28 April 2014

Number of times this article has been viewed

\section{Irmina Urbanek \\ Krzysztof Kaczmarek \\ Iwona Cygankiewicz \\ Pawel Ptaszynski}

Department of Electrocardiology,

Chair of Cardiology and

Cardiosurgery, Medical University,

Lodz, Poland
Correspondence: Pawel Ptaszynski Department of Electrocardiology, Chair of Cardiology and Cardiosurgery, Medical University Lodz, Regional Sterling Heart Disease Center, Sterling I/3, 91-425 Lodz, Poland

$\mathrm{Tel}+48426644304$

Fax +48 426644269

Email pawel_ptaszynski@wp.pl

\begin{abstract}
Heart rate is not only a major risk marker in heart failure but also a general risk marker. Within the last few years, it has been demonstrated that reduction of resting heart rate to $<70$ bpm is of significant benefit for patients with heart failure, especially those with impaired left ventricular systolic function. Ivabradine is the first innovative drug synthesized to reduce heart rate. It selectively and specifically inhibits the pacemaker $I_{f}$ ionic current, which reduces cardiac pacemaker activity. Therefore, the main effect of ivabradine therapy is a substantial lowering of heart rate. Ivabradine does not influence intracardiac conduction, contractility, or ventricular repolarization. According to the European Society of Cardiology guidelines, ivabradine should be considered in symptomatic patients (New York Heart Association functional class II-IV) with sinus rhythm, left ventricular ejection fraction $\leq 35 \%$, and heart rate $\geq 70 \mathrm{bpm}$ despite optimal treatment with a beta-blocker, angiotensin-converting enzyme inhibitor/angiotensin receptor blocker, and a mineralocorticoid receptor antagonist. As shown in numerous clinical studies, ivabradine improves clinical outcomes and quality of life and reduces the risk of death from heart failure or cardiovascular causes. Treatment with ivabradine is very well tolerated and safe, even at maximal recommended doses.
\end{abstract}

Keywords: ivabradine, heart failure, heart rate

\section{Introduction}

Heart failure, particularly in patients with impaired left ventricular systolic function, represents a major therapeutic challenge in modern cardiology. Despite advances in pharmacotherapy and widespread use of highly specialized devices, such as cardioverter defibrillators and pacemakers with a cardiac resynchronization function, heart failure remains a progressive and incurable disease. Therefore, the main objectives of new therapeutic strategies are to inhibit disease progression and to reduce the incidence of rehospitalization and total mortality. Inhibition of neurohumoral activation, which contributes to development and perpetuation of heart failure, remains the major goal of pharmacological strategies. According to the European Society of Cardiology guidelines, at least three neurohumoral antagonists, ie, an angiotensin-converting enzyme inhibitor (or angiotensin receptor blocker), a beta-blocker, and a mineralocorticoid receptor antagonist, should be considered in every patient with heart failure and reduced ejection fraction. These agents play a fundamental role in modifying the course of systolic heart failure and are commonly used in conjunction with diuretics to relieve symptoms and signs of congestion. ${ }^{1}$

Epidemiological observations have shown that a heart rate $>85 \mathrm{bpm}$ is associated with a greater risk of cardiovascular events and higher all-cause mortality. ${ }^{2,3}$ Recently 
published studies clearly document that a reduction in resting heart rate to $<70 \mathrm{bpm}$ provides significant benefit for patients with heart failure, especially those with impaired left ventricular systolic function. Several types of drugs, including beta-blockers, calcium channel antagonists, digoxin, and amiodarone, can be used for reduction of heart rate. Unfortunately, all of these agents have cardiovascular effects other than rate reduction, as well as significant side effects.

The most recently published version of the European Society of Cardiology guidelines for the management of heart failure and reduced ejection fraction introduced ivabradine as a therapeutic option. This novel $\mathrm{I}_{f}$ channel blocker should be administered in symptomatic patients (New York Heart Association functional class [NYHA] II-IV) with left ventricular ejection fraction (LVEF) $\leq 35 \%$, in whom heart rate $\geq 70$ bpm is still observed on optimal doses of an angiotensinconverting enzyme inhibitor (or angiotensin receptor blocker in the event of intolerance), a beta-blocker, and diuretics. ${ }^{1}$ This new recommendation adapted the positive results of two clinical trials, ie, SHIFT (Systolic heart failure treatment with the $\mathrm{I}_{f}$ inhibitor ivabradine trial) and BEAUTIFUL (Morbidity-mortality evaluation of the $\mathrm{I}_{f}$ inhibitor ivabradine in patients with coronary artery disease and left ventricular dysfunction). ${ }^{4,5}$

\section{Pharmacology, mode of action, and pharmacokinetics}

Ivabradine is the first modern selective drug synthesized with the intention of reducing heart rate. It is an organic compound used in the form of a hydrochloride (Figure 1). Ivabradine selectively and specifically inhibits the pacemaker $\mathrm{I}_{f}$ ionic current in a dose-dependent manner. The $f$ stands for "funny" due to its unique properties compared with other systems known at the time of its discovery. $\mathrm{I}_{f}$ is a hyperpolarization-activated $\mathrm{Na}^{+} / \mathrm{K}^{+}$inward channel regulated by the autonomic nervous system. This current controls spontaneous depolarization in the sinus node during relaxation of the heart and thus regulates heart rate. Inhibition of this channel reduces cardiac pacemaker activity and slows heart rate. ${ }^{6,7}$ The $\mathrm{I}_{f}$ current is highly expressed in the sinoatrial node and is one of the most important ionic currents regulating natural pacemaker activity. Taking into consideration the above-mentioned properties, in particular its lack of effect on conduction in the atria, atrioventricular node, and ventricles, as well as its lack of effect on cardiac contractility, ivabradine seems to be a promising treatment option for patients with heart failure.

Ivabradine in recommended doses reduces heart rate by approximately $10 \mathrm{bpm}$, which leads to a reduction in cardiac
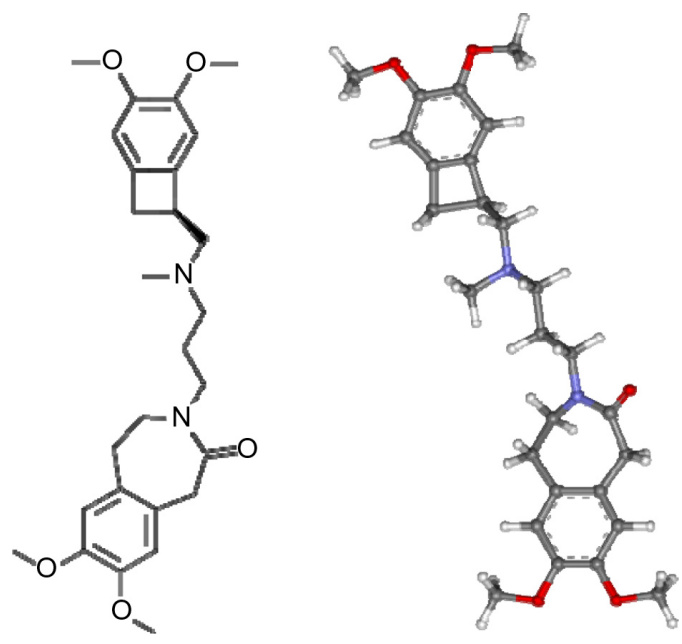

Figure I Chemical structure of ivabradine.

workload and oxygen consumption by cardiac muscle. The heart rate decreases almost linearly with increasing doses of ivabradine up to $15-20 \mathrm{mg}$ twice daily. At higher doses, there is a tendency for these effects to plateau. Ivabradine may have an impact on $\mathrm{I}_{h}$ channels in the retina, which are very similar in structure to cardiac $\mathrm{I}_{f}$ channels. This current participates in temporal resolution of the visual system by curtailing the retinal response to bright light stimuli. Under certain circumstances, partial inhibition of $\mathrm{I}_{h}$ by ivabradine may cause visual impairment. Approximately $14.5 \%$ of patients taking ivabradine experience luminous phenomena, described as sensations of enhanced brightness in a fully maintained visual field. These symptoms are transient and fully reversible. In clinical studies, about $1 \%$ of patients had to discontinue the drug because of such sensations, which generally occurred within the first 2 months of treatment. ${ }^{8}$ Other common adverse reactions include symptomatic bradycardia (in $2 \%-5 \%$ of patients), headaches $(2.6 \%-4.8 \%){ }^{8}$ ventricular extrasystoles, and dizziness.

Ivabradine is rapidly and almost completely absorbed from the gastrointestinal tract after oral administration. In the fasted state, peak concentrations are reached in 1 hour after dosing. Bioavailability is about $40 \%$ due to the first-pass effect in the intestine and liver. Food intake delays absorption by about 1 hour, and increases exposure to the drug in plasma by about $20 \%-30 \%$. Ivabradine is $70 \%$ protein-bound, and is metabolized predominantly in the liver and intestine by oxidation via cytochrome P450 (CYP)3A4. Therefore, potent inhibitors or inducers of CYP3A4 may have a significant effect on the plasma concentration of ivabradine. The concentration of active metabolites is approximately $20 \%$ higher in patients with moderate hepatic impairment than in 
those with normal liver function. Metabolites are excreted in a similar proportion in feces and urine. Approximately $4 \%$ of the dose is excreted unchanged in the urine. Age does not affect the pharmacokinetics of the drug.

\section{Ivabradine in the treatment of chronic heart failure}

Over the last few years, heart rate has become not only a prognostic indicator but also a goal of optimal therapy. The research interest in modulation of heart rate was initiated by Levine. ${ }^{9}$ This original hypothesis was supported by observations of the relationship between life expectancy and the frequency of heart muscle contraction in a number of living organisms. Experimental and clinical data suggest that sustained tachycardia contributes to the pathogenesis of vascular disease. In animal studies, accelerated heart rate is associated with cellular signaling events and leads to vascular oxidative stress, endothelial dysfunction, and acceleration of atherogenesis. Clinical studies have also reported a positive correlation between increased resting heart rate and circulating markers of inflammation. ${ }^{10}$

Early studies of ivabradine, such as INITIATIVE (International trial on the treatment of angina with ivabradine versus atenolol $)^{8}$ and BEAUTIFUL, ${ }^{5}$ focused on patients with stable coronary artery disease. Pathophysiological evidence showed that selective reduction of heart rate improves blood flow and distribution in ischemic myocardium. This effect is associated with proportional improvement in contractile function of ischemic myocardium. In contrast with $\beta$-blockers, selective reduction of heart rate improves both flow and function but has no residual negative effects, such as unmasked $\alpha$-adrenergic coronary vasoconstriction or negative inotropic action. ${ }^{11}$

INITIATIVE compared the efficacy of ivabradine at increasing doses from $5 \mathrm{mg}$ to $10 \mathrm{mg}$ over 16 weeks with that of atenolol at increasing doses from $50 \mathrm{mg}$ to $100 \mathrm{mg} .{ }^{8}$ The reduction in heart rate at peak exercise test was greater on atenolol (14 bpm) than on ivabradine (8.6-10.3 bpm for doses of 7.5-10 mg). Further, both treatment options were comparable for most parameters measured, including time to onset of angina and time to onset of ST segment depression in M1 (5 mg ivabradine versus $50 \mathrm{mg}$ of atenolol). The number of episodes of angina pectoris was reduced by two thirds in both groups of patients.

BEAUTIFUL was a randomized, double-blind, placebocontrolled study ${ }^{3}$ in which ivabradine was compared with placebo in 10,917 patients with stable coronary artery disease and left ventricular dysfunction (LVEF <40\%). Median follow-up was 19 months. No significant reduction in the primary composite end point (death from cardiovascular causes, hospitalization for acute myocardial infarction, and hospitalization for heart failure) was observed. However, in patients with a baseline heart rate $\geq 70 \mathrm{bpm}$, ivabradine significantly reduced the risk of hospitalization for fatal and nonfatal myocardial infarction by $36 \%(P=0.001)$, the risk of coronary revascularization by $30 \%(P=0.016)$, and coronary events by $22 \%(P=0.023)$. A post hoc analysis of the effects of ivabradine in BEAUTIFUL, focused on a subgroup of patients with angina as a limiting symptom at baseline, raised the possibility that ivabradine may be helpful for reducing major cardiovascular events in patients with stable coronary artery disease and left ventricular systolic dysfunction with limiting angina. ${ }^{12}$

However, Heusch, ${ }^{13}$ when commenting on the BEAUTIFUL trial, focused on its original findings, ie, that the benefits of ivabradine derive from its ability to protect against ischemia and not its ability to reduce heart rate, and that this protection is more pronounced in patients with a heart rate $>70 \mathrm{bpm}$. Further, according to author, the reduction of the heart rate in the ivabradine group by only $7 \mathrm{bpm}$ more than in the placebo group seems to be too insignificant to bind them with the profound protection in heart failure. Quoting experimental studies in pigs that reported a significant reduction in infarct size with ivabradine, not only when given before ischemia but also when given just after reperfusion, and that this reduction in infarct size was largely preserved when the reduction in heart rate was offset by atrial pacing, Heusch hypothesized that ivabradine has a pleiotropic protective action beyond its bradycardic effect. The mechanism(s) underlying this pleiotropic protective effect have been suggested to include attenuation of damage by reactive oxygen species and reduced sodium influx through the $\mathrm{I}_{f}$ current, with secondary reduction of sodium-calcium exchange and an ultimate reduction in calcium overload. ${ }^{13}$

SHIFT $^{14}$ investigated ivabradine in patients with heart failure, and confirmed that an elevated heart rate increases the risk of atherosclerosis, myocardial infarction, vascular remodeling, and myocardial infarction, and also increases the risk of adverse cardiovascular events in patients with advanced heart failure. This randomized controlled doubleblind clinical trial included 6,505 patients from 37 countries. Its main inclusion criteria were age $\geq 18$ years, sinus rhythm, resting heart rate $\geq 70 \mathrm{bpm}$ for at least 5 minutes during two consecutive visits, stable symptomatic chronic heart failure (NYHA functional class II-IV) of ischemic or nonischemic etiology, hospitalization for worsening heart 
failure within the previous 12 months, and LVEF $\leq 35 \%$. Because of the mechanism of action of ivabradine, patients with atrial fibrillation and those with pacemaker implantation were excluded. The aim of SHIFT was to determine whether addition of ivabradine to optimal medical therapy in accordance with international guidelines reduces the risk of cardiovascular events in patients with moderate or severe heart failure, reduced left ventricular ejection fraction, and heart rate $>70 \mathrm{bpm}$. The initial dose of ivabradine was $5 \mathrm{mg}$ twice daily, and was then increased to the target dose of $7.5 \mathrm{mg}$ twice daily. In the event of symptomatic bradycardia or a resting heart rate $<50 \mathrm{bpm}$ during the titration period, the dose of ivabradine was reduced to $2.5 \mathrm{mg}$ twice daily.

SHIFT confirmed that ivabradine reduces heart rate in an efficient manner. At 28 days of follow-up, placebo-corrected heart rate decreased by 10.9 bpm in patients on ivabradine; at 1 year, the placebo-corrected reduction in heart rate was $9.1 \mathrm{bpm}$, and at the end of the study was $8.1 \mathrm{bpm}$ (Figure 2). ${ }^{4}$

This study showed also that ivabradine reduced the risk of death from heart failure by $26 \%(P=0.014)$ and the risk of hospitalization for heart failure by $26 \%(P<0.0001)$. This result applied to all patients regardless of predefined subgroup, ie, male or female, with or without beta-blocker therapy at randomization, age under or over 65 years, ischemic or nonischemic heart failure, NYHA functional class II, III, or IV, with or without diabetes mellitus, and with or without hypertension. In the subgroup of patients with a baseline heart rate $>75 \mathrm{bpm}$, there was a significant reduction in risk of death from cardiovascular causes of $17 \%$ $(P=0.0166)$ and a decrease in the overall mortality rate of $17 \%(P=0.0109){ }^{4}$

SHIFT also showed that administration of ivabradine in patients with heart failure significantly reduced the risk of the primary composite end point (ie, hospitalization for worsening heart failure or cardiovascular death) by $18 \%$ $(P<0.0001)$ compared with placebo (Figure 3$)$. These benefits were observed after 3 months of treatment. ${ }^{15}$

There have been several subanalyses of the SHIFT data. One showed that ivabradine decreased the total number of hospital admissions due to heart failure by $25 \%$ and also significantly reduced the likelihood of a second or third hospitalization for heart failure during a median 22.9 months of follow-up. Similar observations were made for all-cause and cardiovascular hospitalizations. This study confirms that the benefit of ivabradine therapy is sustained in patients with heart failure. By reducing the overall burden of heart failure admissions, ivabradine can improve quality of life and reduce the health care costs arising from the condition. ${ }^{16}$

Another subanalysis of SHIFT focused on correlations between ivabradine and other drugs commonly used in the treatment of heart failure. A subanalysis by Swedberg et al ${ }^{17}$ addressed the interaction between ivabradine and betablockers, and showed that the effect of ivabradine is driven

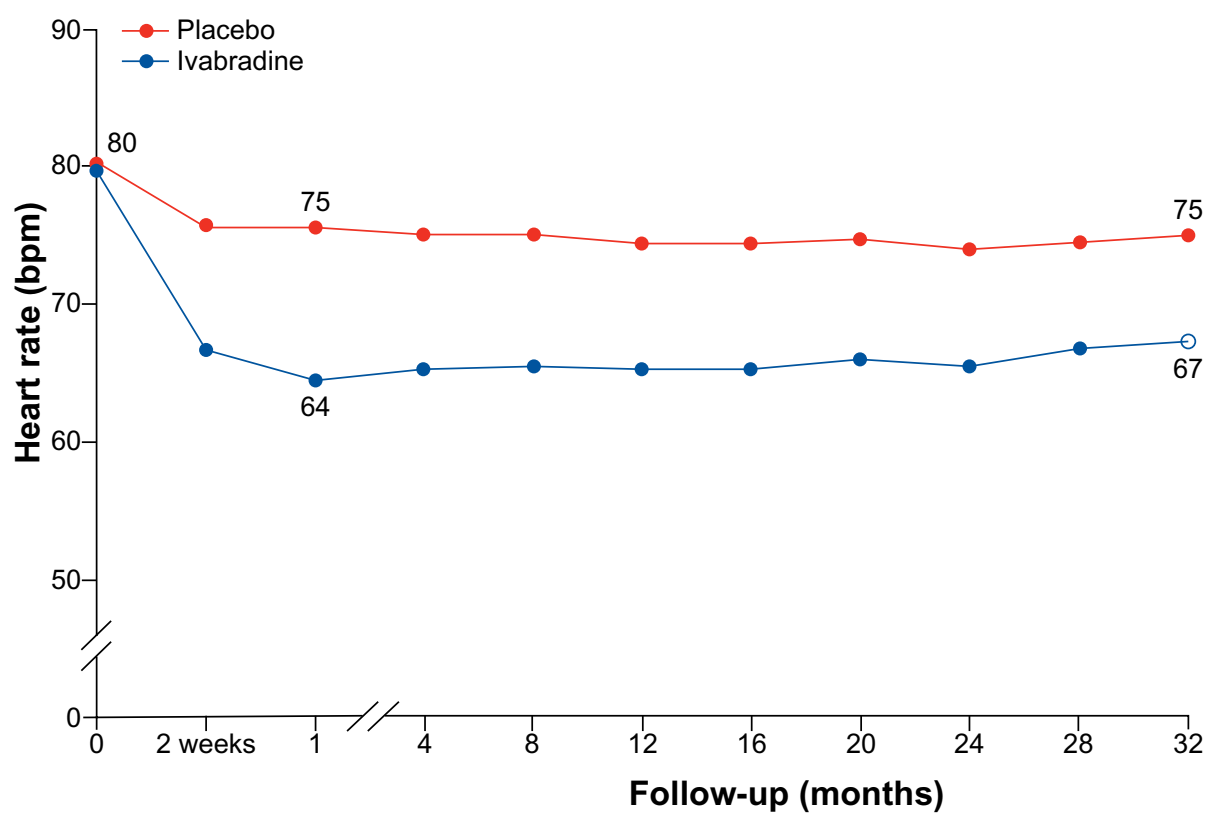

Figure 2 Average heart rate reduction in the ivabradine and placebo groups in the SHIFT trial.

Note: Reprinted from The Lancet, 376(9744), Swedberg K, Komajda M, Böhm M, et al., Ivabradine and outcomes in chronic heart failure (SHIFT): a randomised placebocontrolled study. 875-885, Copyright 2010, with permission from Elsevier. ${ }^{4}$

Abbreviation: SHIFT, Systolic heart failure treatment with the $\mathrm{I}_{f}$ inhibitor ivabradine trial. 
mainly by baseline heart rate and its reduction. Another subanalysis of the SHIFT data ${ }^{18}$ demonstrated that ivabradine and a mineralocorticoid receptor antagonist (MCRA) are complementary. Evaluation of the impact of ivabradine on outcomes, in particular the primary composite endpoint, showed consistency in MCRA versus non - MCRA patients. Especially, the addition of ivabradine should be considered in patients with a heart rate $>70 \mathrm{bpm}$ despite being on a mineralocorticoid receptor antagonist, in order to improve the clinical outcome further.

In another trial, Bagriy et al studied early addition of ivabradine to carvedilol therapy in patients with chronic heart failure. ${ }^{19}$ This study included 41 patients with chronic heart failure (NYHA functional class II-III), a history of previous myocardial infarction, and in sinus rhythm with a heart rate $\geq 70 \mathrm{bpm}$, who had not taken ivabradine or a beta-blocker for at least 2 months. Patients were randomized to receive carvedilol alone or carvedilol and ivabradine. The target dose of carvedilol was $25 \mathrm{mg}$ twice daily. The most common reasons for not reaching the target dose of carvedilol were hypotension, dizziness, and worsening of chronic heart failure. Ivabradine was administered 1-2 days after carvedilol, using an initial dose of $5 \mathrm{mg}$ twice daily titrated up to $7.5 \mathrm{mg}$ twice daily after 1 month if heart rate was still $\geq 70 \mathrm{bpm}$. After 3 months, combined therapy resulted in a shorter time required for uptitration of the beta-blocker $(1.9 \pm 0.5$ months versus $2.7 \pm 0.7$ months), a higher final beta-blocker

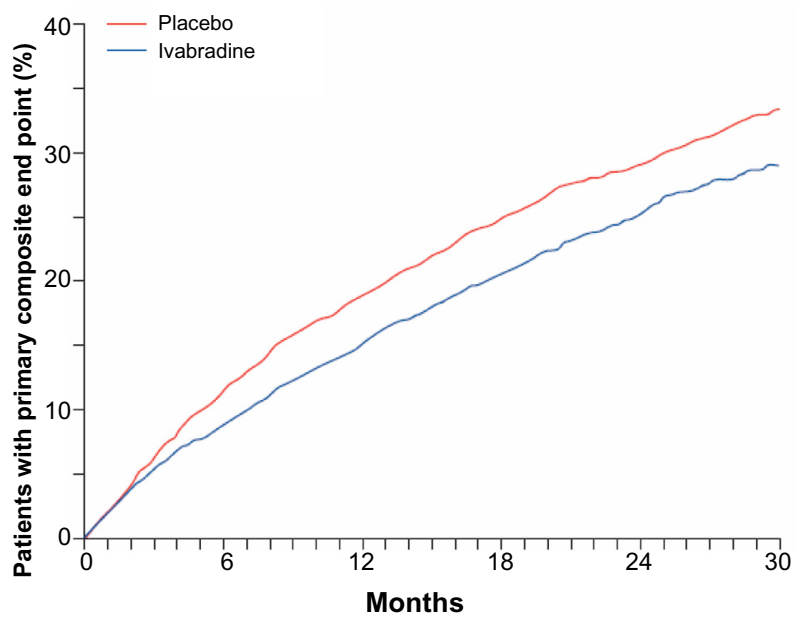

Figure 3 Primary composite end point (hospitalization for worsening heart failure or cardiovascular death) in SHIFT.

Note: Reprinted from The Lancet, 376(9744), Swedberg K, Komajda M, Böhm M, etal., Ivabradine and outcomes in chronic heart failure (SHIFT): a randomised placebocontrolled study. 875-885, Copyright 2010, with permission from Elsevier. ${ }^{4}$

Abbreviation: SHIFT, Systolic heart failure treatment with the $\mathrm{I}_{f}$ inhibitor ivabradine trial. dose $(37.4 \pm 8.4 \mathrm{mg} /$ day versus $29.6 \pm 6.2 \mathrm{mg} /$ day), greater heart rate reduction $(12.9 \pm 3.5 \mathrm{bpm}$ versus $7.2 \pm 2.4 \mathrm{bpm})$, and better exercise capacity (mean change in 6-minute walking test distance, $68.3 \pm 12.7 \mathrm{~m}$ versus $32.4 \pm 11.7 \mathrm{~m}$ ) comparing with carvedilol monotherapy.

Myocardial remodeling plays an important role in the pathophysiology and progression of heart failure. ${ }^{20} \mathrm{An}$ echocardiographic substudy of SHIFT, ${ }^{21}$ including 411 patients, was performed at baseline and 8 months after randomization. Selective heart rate reduction in patients receiving ivabradine led to a significant increase in LVEF by 2.4 points, a reduction in end-systolic volume index, and a decrease in end-diastolic volume index. The change in left ventricular end-systolic volume index was independent of baseline LVEF, etiology of heart failure, or dose of betablocker, and became the basis for further discussion regarding the influence of selective reduction of heart rate on left ventricular remodeling and function.

Sarullo et al evaluated the effect of 3 months of ivabradine therapy on exercise capacity and plasma levels of N-terminal pro-brain natriuretic peptide. ${ }^{22}$ Their study was performed in 60 patients with stable ischemic chronic heart failure, NYHA class II-III disease, and an LVEF of $40 \%$, who were randomized to an ivabradine group and or a control group. After 3 months of treatment, no significant differences were found in the control group, whereas a significant improvement in exercise capacity (from $14.8 \pm 2.5$ minutes to $28.2 \pm 3.5$ minutes, $P<0.0001$ ) and gas exchange (peak oxygen consumption increased from $13.5 \pm 1.3 \mathrm{~mL} / \mathrm{kg}$ per minute to $17.9 \pm 2.4 \mathrm{~mL} / \mathrm{kg}$ per minute, $P<0.0001)$ was noted in the ivabradine group. Oxygen consumption at anaerobic threshold increased (from $11.9 \pm 1.4 \mathrm{~mL} /$ $\mathrm{kg}$ per minute to $15.3 \pm 1.4 \mathrm{~mL} / \mathrm{kg}$ per minute; $P<0.0001)$ and positive neurohormonal modulation ( $\mathrm{N}$-terminal pro-brain natriuretic peptide levels decreased from $2,356 \pm 2,113 \mathrm{pg} / \mathrm{mL}$ to $1,434 \pm 1,273 \mathrm{pg} / \mathrm{mL}$ ) was observed. These positive effects of ivabradine were also associated with an improvement in functional heart failure class and quality of life.

\section{Long-term safety and tolerability issues}

SHIFT demonstrated the excellent safety of ivabradine. Only $1 \%$ of patients had to be withdrawn from the trial due to bradycardia (symptomatic or asymptomatic). Visual side effects occurred in a limited number of patients. ${ }^{23}$

A subanalysis of SHIFT confirmed the benefits of ivabradine in reduction of heart rate and its acceptable profile, independent of baseline clinical status and objective severity of heart failure. ${ }^{24}$ This substudy included 343 
patients in NYHA class IV or with LVEF $\leq 20 \%$ who received ivabradine and 369 patients from the placebo arm of the SHIFT population. Compared with the rest of the SHIFT population (patients with lower NYHA class or higher LVEF), this subgroup had a higher mean heart rate (82.1 bpm versus $79.6 \mathrm{bpm}, P<0.001$ ), lower systolic and diastolic blood pressure (116 mmHg versus $122 \mathrm{mmHg}$ and $73 \mathrm{mmHg}$ versus $76 \mathrm{mmHg}$, respectively, $P<0.001$ ), more coexisting chronic obstructive pulmonary disease (COPD, $15 \%$ versus $11 \%, P=0.002$ ), and more frequent diuretic therapy $(90 \%$ versus $82 \%, P<0.001)$. These patients were also less frequently treated with beta-blockers ( $87 \%$ versus $90 \%, P=0.028$ ) and received smaller doses of these agents. After 1 month of therapy, ivabradine reduced heart rate by $15 \mathrm{bpm}$ in all patients. In both groups, ivabradine reduced the primary composite end point defined as any hospitalization for heart failure or cardiovascular death (hazard ratio [HR] 0.84, 95\% confidence interval [CI] 0.67-1.07 versus HR 0.82, CI 0.74-0.91, respectively; $P=0.854$ for interaction), heart failure hospitalizations (HR 0.83, CI 0.63-1.11 versus HR 0.73 , CI $0.64-0.83 ; P=0.419$ for interaction), and cardiovascular death (HR 0.78, CI 0.58-1.06 versus HR 0.94 , CI $0.82-1.08 ; P=0.264$ for interaction) in a comparable manner. In severely ill patients with a baseline heart rate $\geq 75 \mathrm{bpm}(\mathrm{n}=272)$, ivabradine reduced the primary composite end point by $25 \%(P=0.045)$ and cardiovascular death by $32 \%(P=0.034)$. Symptomatic and asymptomatic bradycardia occurred more frequently in patients taking ivabradine than in the placebo group $(3.5 \%$ versus $0.5 \%$, $P=0.005$; and $5.2 \%$ versus $1.6 \% 0.5, P=0.007$ ) and resulted in permanent withdrawal from the study in $1.2 \%$ of patients on ivabradine and $<1 \%$ of those on placebo $(P=0.202)$. Visual symptoms occurred in $2 \%$ of the ivabradine group versus $<1 \%$ in the placebo group. The incidence of atrial fibrillation was similar on and off ivabradine therapy (10.2\% versus $9.2 \%, P=0.655)$. Thus, it can be concluded that the safety profile of ivabradine in severely ill patients was similar to that of the general patient population in SHIFT, with comparable efficacy.

Another SHIFT trial subanalysis showed that age does not limit appropriate treatment with ivabradine in patients with chronic heart failure and systolic dysfunction. ${ }^{25}$ The efficacy and safety of ivabradine were evaluated in the following age groups: $<53$ years, 53-60 years, 60-69 years, and $\geq 69$ years. In all age groups, ivabradine reduced heart rate to a similar extent (by $11 \mathrm{bpm}$ ), with a reduction in risk of the primary composite end point (by $38 \%$ in patients $<53$ years and by $16 \%$ in patients $\geq 69$ years). As anticipated, the incidence of serious adverse events and side effects leading to treatment withdrawal increased with age, but no substantial differences were noted between the ivabradine and placebo groups. As expected, bradycardia and visual symptoms occurred more frequently in patients on ivabradine, but at a similar rate regardless of age. No severe bradycardia or conduction disturbances were observed.

Based on the study by Majewski et al, ivabradine seems to be a safe and effective drug in patients with asthma or COPD. ${ }^{26}$ This randomized, single-center, double-blind, placebo-controlled, crossover trial included 40 patients (20 with asthma and 20 with COPD). All patients received ivabradine $7.5 \mathrm{mg}$ or placebo twice daily for 5 days in a crossover manner, with a washout of at least 2 days between treatments. Ivabradine significantly reduced mean heart rate when compared with placebo in patients with asthma and in those with COPD $(P<0.001)$. No significant difference was found in morning and evening peak expiratory flow rate, diurnal variability in peak expiratory flow, use of rescue medication, or daily symptom scores between patients treated with ivabradine and those treated with placebo. The treatment was well tolerated. Visual symptoms were reported by $5 \%$ of patients in the ivabradine group. This study shows that ivabradine is an interesting treatment option for heart failure in patients with respiratory disease and/or contraindications to beta-blockers.

\section{Quality of life, patient satisfaction, adherence, and uptake}

In addition to decreasing hospitalization and total mortality in patients with heart failure improving their quality of life should not be forgotten, which sometimes remains in the shadow of clinical studies. Heart failure-related symptoms are correlated with a very low quality of life. The medications commonly recommended for the treatment of heart failure have an influence on health status and improve the prognosis. However, they have minimal (angiotensinconverting enzyme inhibitors) or no (beta-blockers) effect on quality of life in patients with heart failure. A large subanalysis of 1,944 patients in SHIFT used the Kansas City Cardiomyopathy Questionnaire (KCCQ), which is an effective tool for evaluating health status in patients with heart failure. ${ }^{27}$ This questionnaire consists of two separate components, ie, a clinical summary score which evaluates the disability directly related to the disease and an overall summary score which includes social limitation. Patients receiving ivabradine experienced a significant improvement in their overall and clinical summary scores 
after 12 months of therapy. Interestingly, these changes were correlated with a change in heart rate. The relationship was found not only in the active treatment arm but also in the placebo arm, suggesting that heart rate reduction is associated with improved quality of life. ${ }^{23}$

Sarullo et al reported that use of ivabradine improved NYHA functional class, alleviated symptoms, and improved quality of life. ${ }^{22}$ They assessed quality of life at baseline and after 3 months in patients taking ivabradine and in those on placebo using the Minnesota Living with Heart Failure questionnaire. ${ }^{28,29}$ At baseline, there was no significant difference in mean Minnesota Living with Heart Failure score between the ivabradine group (30.9 \pm 2.3$)$ and the control group (30.6 \pm 2.1 ). After 3 months of therapy, Minnesota Living with Heart Failure Questionnaire scores were significantly improved in the ivabradine group $(37.5 \pm 1.9 ; P<0.0001)$ compared with baseline, with no significant change in the control group (31.2 \pm 2.6$).^{22}$

\section{Conclusion}

In accordance with the European Society of Cardiology guidelines, the treatment goals in patients with heart failure are to relieve heart rate-related symptoms, prevent hospital admission, and improve survival. ${ }^{1}$ According to the European Heart Rhythm Association/ Heart Rhythm Society consensus statement, an important objective of medical treatment for heart failure involves lowering heart rate to the lowest tolerated rate above 50 bpm. ${ }^{30}$

All international guidelines recommend beta-blockers as first-line therapy in patients with heart failure and low LVEF. However, these drugs are often difficult to manage in real life, mainly due to side effects and contraindications, ${ }^{31}$ which explains why contemporary surveys show that heart rate remains elevated in many patients on beta-blocker therapy. If a patient cannot tolerate a beta-blocker or if uptitration is ineffective, ivabradine should be considered as a treatment option. As recently documented, ivabradine can be administered in order to improve clinical outcomes, particularly to decrease heart failure hospitalizations, reduce the risk of death from cardiovascular causes, and improve quality of life. Treatment with ivabradine is safe and well tolerated by patients with heart failure. Side effects are not common, and if they occur, they are mild in severity and reversible after reduction in the drug dose.

\section{Disclosure}

The authors report no conflicts of interest in this work.

\section{References}

1. McMurray JJ, Adamopoulos S, Anker SD, et al. ESC guidelines for the diagnosis and treatment of acute and chronic heart failure 2012: The Task Force for the Diagnosis and Treatment of Acute and Chronic Heart Failure 2012 of the European Society of Cardiology. Developed in collaboration with the Heart Failure Association of the ESC. Eur Heart J. 2012;14(8):803-869.

2. Dyer AR, Persky V, Stamler J, et al. Heart rate as a prognostic factor for coronary heart disease and mortality: findings in three Chicago epidemiologic studies. Am J Epidemiol. 1980;112(6):736-749.

3. Kannel WB, Cupples LA. Heart rate and cardiovascular mortality: the Framingham study. Am Heart J. 1987;113(6):1489-1494.

4. Swedberg K, Komajda M, Böhm M, et al. Ivabradine and outcomes in chronic heart failure (SHIFT): a randomised placebo-controlled study. Lancet. 2010;376(9744):875-885.

5. Fox K, Ford I, Steg PG, Tendera M, Robertson M, Ferrari R. Ivabradine for patients with stable coronary artery disease and left-ventricular systolic dysfunction (BEAUTIFUL): a randomized, double-blind, placebo-controlled trial. Lancet. 2008;372(9641):807-816.

6. Thollon C, Cambarrat C, Vian J, Prost JF, Peglion JL, Vilaine JP. Electrophysiological effects of S16257, a novel sino-atrial node modulator, on rabbit and guinea-pig cardiac preparations: comparison with UL-FS 49. Br J Pharmacol. 1994;112(1):37-42.

7. Sulfi S, Timmis AD. Ivabradine - the first selective sinus node I(f) channel inhibitor in the treatment of stable angina. Int J Clin Pract. 2006;60(2):222-228.

8. Tardif JC, Ford I, Tendera M, Bourassa MG, Fox K. Efficacy of ivabradine, a new selective $\mathrm{I}_{\mathrm{f}}$ inhibitor, compared with atenolol in patients with chronic stable angina. Eur Heart J. 2005;26(23):2529-2536.

9. Levine HJ. Rest heart rate and life expectancy. J Am Coll Cardiol. 1997;30(4):1104-1106.

10. Custodis F, Schirmer SH, Baumhäkel M, Heusch G, Böhm M, Laufs U. Vascular pathophysiology in response to increased heart rate. $\mathrm{J} \mathrm{Am} \mathrm{Coll}$ Cardiol. 2010;56(24):1973-1983.

11. Heusch G. Heart rate in the pathophysiology of coronary blood flow and myocardial ischaemia: benefit from selective bradycardic agents Br J Pharmacol. 2008;153(8):1589-1601.

12. Fox K, Ford I, Steg G, Tendera M, Robertson M, Ferrari R. Relationship between ivabradine treatment and cardiovascular outcomes in patients with stable coronary artery disease and left ventricular systolic dysfunction with limiting angina: a subgroup analysis of the randomized, controlled BEAUTIFUL trial. Eur Heart J. 2009;30(19):2337-2345.

13. Heusch G. A BEAUTIFUL lesson-ivabradine protects from ischaemia, but not from heart failure: through heart rate reduction or more? Eur Heart J. 2009;30(19):2300-2301.

14. Böhm M, Swedberg K, Komajda M, et al. HR as a risk factor in chronic heart failure (SHIFT): the association between HR and outcomes in a randomised placebo-controlled trial. Lancet. 2010;376(9744):886-894.

15. Stiles S. SHIFT: adding HR-slowing agent ivabradine to HF meds cuts mortality, hospitalization. Medscape. 2012;33(22):2813-2820. Available from: http://www.medscape.com/viewarticle/790694. Accessed March $12,2014$.

16. Borer JS, Böhm M, Ford I, et al. Effect of ivabradine on recurrent hospitalization for worsening heart failure in patients with chronic systolic heart failure: the SHIFT study. Eur Heart J. 2012;33(22):2813-2820.

17. Swedberg K, Komajda M, Böhm M, et al. Effects on outcomes of HR reduction by ivabradine in patients with CHF: is there an influence of beta-blocker dose? Findings from the SHIFT study. J Am Coll Cardiol. 2012;59(22):1938-1945.

18. Komajda M, Böhm M, Borer J, et al. Influence of background treatment with mineralocorticoid receptor antagonists on ivabradine's effects in patients with chronic heart failure. Eur $J$ Heart Fail. 2013;15(1):79-84. 
19. Bagriy AE, Shchukina EV, Malovichko SI. Early addition of ivabradine shortens carvedilol uptitration and improves exercise capacity in patients with chronic heart failure. European Journal of Heart Failure 2013. 15(S1), S140.

20. Kramer DG, Trikalinos TA, Kent DM, Antonopoulos GV, Konstam MA, Udelson JE. Quantitative evaluation of drug or device effects on ventricular remodeling as predictors of therapeutic effects on mortality in patients with heart failure and reduced ejection fraction: a metaanalytic approach. J Am Coll Cardiol. 2010;56(5):392-406.

21. Tardif JC, O’Meara E, Komajda M, et al. Effects of selective HR reduction with Ivabradine on left ventricular remodeling and function: results from the SHIFT echocardiography substudy. Eur Heart J. 2011;32(20):2507-2515.

22. Sarullo FM, Fazio G, Puccio D, et al. Impact of "off-label" use of ivabradine on exercise capacity, gas exchange, functional class, quality of life and neurohormonal modulation in patients with ischemic chronic heart failure. J Cardiovasc Pharmacol Ther. 2010;15(4):349-355.

23. Komajda M. [Novel approaches to HR modulation in chronic heart failure]. Kardiol Pol. 2013;71(6):549-551. Polish.

24. Borer JS, Bohm M, Ford I, et al. Efficacy and safety of ivabradine in patients with severe chronic systolic heart failure/left ventricular dysfunction in the SHIFT trial. Eur J Heart Fail. 2014;113(3):497-503.

25. Tavazzi L, Swedberg K, Komajda M, et al. Efficacy and safety of ivabradine in chronic heart failure across the age spectrum: insights from the SHIFT study. Eur J Heart Fail. 2013;15(11):1296-1303.
26. Majewski S, Slomka S, Zielinska-WyderkiewiczE, Ciebieada M, Gorski P. Heart rate-lowering efficacy and respiratory safety of ivabradine in patients with obstructive airway disease. Am J Cardiovasc Drugs. 2012;12(3):179-188.

27. Green CP, Porter CB, Bresnahan DR, Spertus JA. Development and evaluation of the Kansas City Cardiomyopathy Questionnaire: a new health status measure for heart failure. J Am Coll Cardiol. 2000;35(5):1245-1255.

28. Belardinelli R, Georgiou D, Cianci G, Purcaro A. Randomized controlled trial of long-term moderate exercise training in chronic heart failure. Effect on functional capacity, quality of life, and clinical outcome. Circulation. 1999;99(9):1173-1182.

29. Hubo SH, Gollub S, Buorge R, et al. Beneficial effect of pimobendan on exercise tolerance and quality of life in patients with heart failure: results of a multicenter trial. Circulation. 1992;85(3):942-949.

30. Daubert JC, Saxon L, Adamson PB, et al. 2012 EHRA/HRS expert consensus statement on cardiac resynchronization therapy in heart failure: implant and follow-up recommendations and management. Europace. 2012;14(9):1236-1286.

31. Maggioni AP, Anker SD, Dahlstrőm U, et al. Are hospitalized or ambulatory patients with heart failure treated in accordance with European Society of Cardiology guidelines? Evidence from 12440 patients of the ESC Heart Failure Long-Term Registry. Eur $J$ Heart Fail. 2013;15(10):1173-1184.
Drug, Healthcare and Patient Safety

\section{Publish your work in this journal}

Drug, Healthcare and Patient Safety is an international, peer-reviewed open-access journal exploring patient safety issues in the healthcare continuum from diagnostic and screening interventions through to treatment, drug therapy and surgery. The journal is characterized by the rapid reporting of reviews, original research, clinical, epidemiological and

\section{Dovepress}

post-marketing surveillance studies, risk management, health literacy and educational programs across all areas of healthcare delivery. The manuscript management system is completely online and includes a very quick and fair peer-review system. Visit http://www.dovepress.com/ testimonials.php to read real quotes from published authors. 